\title{
FATORES ASSOCIADOS A EMERGENCIAS MÉDICAS DOMICILIARES EM IDOSOS BRASILEIROS
}

\author{
Camila Zanesco ${ }^{1}$ \\ Danielle Bordin² \\ Celso Bilynkievycz dos Santos ${ }^{3}$ \\ Cristina Berger Fadel ${ }^{4}$
}

\section{resumo}

O objetivo do estudo consiste em analisar os fatores associados à vivência de emergências domiciliares por idosos brasileiros. Estudo de caráter transversal, quantitativo, com fonte de dados secundária - Pesquisa Nacional de Saúde - 2013. A amostra foi composta por indivíduos com 60 anos ou mais ( $n=23.815$ ). A variável desfecho foi nomeada "Emergência domiciliar" e foram incluídas 56 variáveis independentes. Os dados foram tratados, e posteriormente foi realizada redução de dimensionalidade no software Waikato Environment for Knowledge Analysis. As variáveis fortemente associadas com situações de emergência domiciliar foram analisadas através da regressão logística para mensurar a intensidade da ligação. Os achados apontam

\footnotetext{
1 Enfermeira. Mestranda pelo Programa de Pós-Graduação Stricto Sensu em Ciências da Saúde da Universidade Estadual de Ponta Grossa, Brasil - PR. E-mail: camila_zanesco@hotmail.com.

2 Cirurgiã-dentista. Doutora em Odontologia Social e Preventiva. Docente colaboradora do Departamento de Enfermagem e Saúde Pública, Universidade Estadual de Ponta Grossa, Brasil - PR. E-mail: daniellebordin@hotmail.com.

3 Cientista da computação. Doutor em Engenharia de Produção. Técnico em Assuntos Universitários, Departamento de Odontologia, Universidade Estadual de Ponta Grossa, Brasil - PR. E-mail: bilynkievycz@globo.com.

4 Cirurgiã-dentista. Doutora em Odontologia Social e Preventiva. Docente adjunta do Departamento Odontologia, Universidade Estadual de Ponta Grossa, Brasil - PR. E-mail: cbfadel@gmail.com.
} 
que as situações de emergência domiciliar envolvendo idosos têm maior probabilidade de acontecer quando o indivíduo precisou ser internado nos últimos 12 meses $(\mathrm{OR}=7,02)$, teve dificuldade em ir ao médico sozinho $(O R=2,10)$, autopercebeu-se em situação negativa de saúde $(O R=2,00)$, teve dificuldade em administrar as próprias finanças $(O R=1,71)$, teve dificuldade de sentar-se ou levantar-se de uma cadeira $(O R=1,67)$, deixou de realizar qualquer atividade habitual por motivo de saúde $(\mathrm{OR}=1,66)$, procurou o mesmo médico ou serviço de saúde quando precisou de atendimento $(\mathrm{OR}=1,34)$ e teve frequência superior a quatro consultas médicas nos últimos 12 meses $(\mathrm{OR}=1,39)$. A interpretação dos presentes achados compõe uma importante base para o planejamento e desenvolvimento de ações preventivas e promotoras de saúde.

palavras-chave

Sistemas de Saúde. Saúde do Idoso. Emergências.

\section{Introdução}

O aumento do número de indivíduos com 60 ou mais anos, quando analisado sob a ótica da epidemiologia e dos serviços de saúde, acarreta preocupações e dificuldades sócio-organizacionais frente às inúmeras demandas geradas no âmbito da integralidade à saúde. Considerando ainda o perfil epidemiológico da morbimortalidade desses indivíduos, as complicações ganham maior destaque e alcançam amplitude mundial (NUNES et al., 2017; BRITO; MENEZES; OLINDA, 2016; FIALHO et al., 2014; ALMEIDA et al., 2017). As necessidades em saúde da população idosa derivam especialmente do processo natural e progressivo relacionado à diminuição da capacidade funcional e cognitiva, o qual pode ser acelerado devido à presença de doenças (PASKULIN; VALER; VIANNA, 2011; NUNES et al., 2017; BRITO; MENEZES; OLINDA, 2016; FIALHO et al., 2014).

Os gastos decorrentes dessa realidade social e epidemiológica sobrecarregam de forma excessiva recursos públicos e privados, principalmente no campo da saúde (NUNES et al., 2017; FIALHO et al., 2014; SOARES et al., 2016). Ainda, além de gerarem instabilidade na economia mundial, repercutem no meio familiar e da sociedade (CALDAS et al., 2015; SOARES et al., 2016). Dessa forma, a realidade retratada requer qualidade, agilidade e resolutividade por parte dos serviços de saúde, nos diversos níveis do sistema (CALDAS et al., 2015; 
COSTA; CIOSAK, 2010), considerando, além da assistência, o cuidado e o apoio aos indivíduos e suas famílias.

Outra imponente questão, responsável por inúmeras preocupações por parte da população idosa, bem como dos gestores de serviços de saúde, são as emergências médicas domiciliares, as quais requerem reconhecimento e cuidados imediatos, por se tratar de situações que expõem o indivíduo ao risco de perda de órgãos, membros e da própria vida (CALDAS et al., 2015; HIRSHON et al., 2013). Compreender os primórdios dos desfechos negativos relacionados ao aspecto em debate faz-se indispensável, visto que o conhecimento viabiliza o planejamento e a atuação focada nas reais demandas e possibilidades da atenção em saúde (BONATO, 2011; COSTA; CIOSAK, 2010). Nessa perspectiva, dispondo da magnitude e relevância empregada aos inquéritos de base populacional, como é o caso da Pesquisa Nacional de Saúde (PNS), a qual se constitui uma profícua fonte de dados (SZWARCWALD et al., 2014; SOUZA-JÚNIOR et al., 2015; DAMACENA et al., 2015; JEREZ-ROIG et al., 2016), objetiva-se, por meio do presente estudo, conhecer os fatores associados a emergências médicas domiciliares de idosos brasileiros.

\section{Metodologia}

Constitui-se como estudo transversal, quantitativo, o qual utilizou dados originários do inquérito de base populacional, a PNS - 2013, idealizada pelo Ministério da Saúde e conduzida pelo Instituto Brasileiro de Geografia e Estatística (IBGE) (2014), foi aprovada pela Comissão Nacional de Ética em Pesquisa para Seres Humanos, do Ministério da Saúde, sob o Parecer n.ำ 328.159/2013. A PNS - 2013 é uma pesquisa de base domiciliar que utilizou como plano amostral uma amostragem probabilística oriunda de conglomerados, subdivida em três etapas interligadas: setores censitários, os domicílios e os moradores com 18 anos ou mais (IBGE, 2014).

O nível de precisão almejado para as estimativas dos indicadores de interesse pautou a definição do tamanho da amostragem, totalizando 205.546 indivíduos residentes em 60.900 domicílios pesquisados (IBGE, 2014; SOUZA-JÚNIOR et al., 2015; SZWARCWALD et al., 2014). O relatório da PNS - 2013 expõe de forma detalhada o processo de amostragem e ponderação (IBGE, 2014).

Toda a etapa de coleta de dados foi desempenhada por pesquisadores previamente capacitados e calibrados. Foram utilizados três formulários para guiar a entrevista: o domiciliar, condizente às características do domicílio; um correspondente a todos os moradores do domicílio; e um individual, o qual 
poderia ser respondido para todos os moradores do domicílio por um indivíduo maior de 18 anos de idade, elencado através de sorteio (IBGE, 2014). No estudo em questão, os dados utilizados foram os referentes aos participantes idosos $(\mathrm{n}=23.815)$, derivados dos últimos dois formulários.

A variável desfecho "emergência domiciliar" é resultante da questão "Nos últimos 12 meses, teve atendimento de emergência no domicílio?", tendo como opções para resposta "sim" e "não". Na fase de pré-exploração dos dados, foram elencadas 56 variáveis de interesse para compor o rol de variáveis independentes relacionadas a características sociodemográficas, limitações e adoecimento, dificuldades ao realizar atividades básicas e instrumentais de vida diária, doenças crônicas, utilização de serviços de saúde, internações e hábitos (Tabela 1). Todas as variáveis selecionadas passaram por tratamento, as numéricas foram transformadas em categóricas, algumas variáveis foram recategorizadas e outras dicotomizadas, conforme o preconizado na literatura. Além disso, devido ao desbalanceamento das classes da variável desfecho, realizou-se, para cada variável independente, o balanceamento das classes a partir da variável desfecho, através do método supervisionado do filtro Resample do Waikato Environment for Knowledge Analysis (WEKA) (WITTEN et al., 2016), com vistas a minimizar possíveis vieses dos resultados.

Tabela 1 - Descrição das variáveis independentes usadas no estudo.

\begin{tabular}{ll}
\hline Características sociodemográficas & \\
\hline Sexo & Estado civil \\
ldade & Alfabetização \\
Cor & Nível de educação \\
Vivência com cônjuge & Renda \\
\hline Limitações & \\
\hline Autopercepção da condição de saúde geral & Esteve acamado \\
$\begin{array}{ll}\text { Presença de doença crônica, física ou mental, } \\
\text { limita de alguma forma suas atividades habituais }\end{array}$ & Número de dias acamado \\
$\begin{array}{ll}\text { Deixou de realizar quaisquer de suas atividades } \\
\text { habituais por motivo de saúde }\end{array}$ & Dificuldade de locomoção \\
$\begin{array}{l}\text { Dias que deixou de realizar suas atividades } \\
\text { habituais, por motivo de saúde }\end{array}$ & Dificuldade para enxergar \\
\hline
\end{tabular}




\section{Dificuldades ao realizar Atividades Básicas de Vida Diária}

Presença de dificuldade para comer sozinho com um prato colocado à sua frente, incluindo segurar um garfo, cortar alimentos e beber em um copo

Presença de dificuldade para ir ao banheiro sozinho incluindo sentar e levantar do vaso sanitário

Presença de dificuldade para se vestir sozinho

Presença de dificuldade para sentar ou levantar da cadeira sozinho
Presença de dificuldade para tomar banho sozinho incluindo entrar e sair do chuveiro ou banheira

Presença de dificuldade para andar em casa sozinho de um cômodo a outro da casa

Presença de dificuldade para deitar ou levantar da cama sozinho

\section{Dificuldades ao realizar Atividades Instrumentais de Vida Diária}

Presença de dificuldade para fazer compras sozinho

Presença de dificuldade para tomar os remédios sozinho

Presença de dificuldade para sair sozinho utilizando um transporte
Presença de dificuldade para administrar as finanças sozinho

Presença de dificuldade para ir ao médico sozinho

Presença de dificuldade para administrar as finanças sozinho (cuidar do seu próprio dinheiro)

\section{Doenças crônicas}

Presença de alguma doença crônica, física ou mental

Diabetes

Hipertensão

Colesterol elevado

Acidente vascular cerebral ou derrame

Problema crônico na coluna
Distúrbio osteomuscular relacionado ao trabalho

Artrite

Depressão

Doença pulmonar obstrutiva crônica

Câncer

Problema renal crônico

\section{Utilização de serviços de saúde}

Local que costuma procurar atendimento quando está doente

Tempo desde a última consulta médica

Procura por algum lugar, serviço ou profissional de saúde para atendimento relacionado à própria saúde nas últimas duas semanas

Teve medicamentos receitados na última consulta
Motivo pela procura do atendimento

Local onde procurou o último atendimento de saúde

Utilizou alguma prática integrativa e complementar, isto é, tratamento como acupuntura, homeopatia, plantas medicinais e fitoterapia etc.

Procura o mesmo lugar, mesmo médico ou mesmo serviço de saúde quando precisa de atendimento de saúde 
Internações

Internação no último ano

Motivo da internação

Número de internações no último ano

Tempo internado

Hábitos

Consumo de bebidas alcoólicas

Exerce atividade física

Faz uso de tabaco

Fonte: Elaborada pelos autores.

Posteriormente, os dados foram alocados em base de dados contendo as variáveis de interesse (Tabela 1), realizou-se o teste de redução de dimensionalidade, utilizando o algoritmo Correlation-based Feature Selection (CFS) (WITTEN et al., 2016), pautando-se no método nomeado como "validação cruzada de 10 Fold". O referido teste identifica simultaneamente as variáveis independentes com elevada relação com a variável dependente e reduzida relação entre si, excluindo possíveis relações de confundimento e permitindo, desse modo, validar relações puras e estritas das variáveis independentes com a variável desfecho, de maneira precisa e isentando ao máximo a interferência humana no processo de identificação das relações, diferentemente de outros testes comumente utilizados na literatura (WITTEN et al., 2016).

Posteriormente, utilizando o software WEKA, as variáveis independentes que tivessem relação com a variável desfecho (emergência domiciliar) foram submetidas à avaliação através da regressão logística, visando à mensuração da intensidade das associações. O modelo formado teve capacidade explicativa de $76,3 \%$ (WITTEN et al., 2016).

\section{Resultados}

A análise descritiva mostra que cerca de 3\% dos idosos investigados tiveram alguma emergência médica no domicílio, considerando o período dos 12 meses que antecederam a coleta. Das 56 variáveis selecionadas (Tabela 1), apenas 8 tiveram forte relação com a variável desfecho, a saber: "autopercepção da condição de saúde geral"; "deixou de realizar quaisquer de suas atividades habituais por motivo de saúde"; "dificuldade para administrar as finanças, ir ao médico e sentar ou levantar da cadeira sozinho"; "procura o mesmo lugar, mesmo médico ou mesmo serviço de saúde quando precisa de atendimento de saúde"; "internação no último ano"; "frequência de consulta no último ano". A análise descritiva destas variáveis e as razões de chances, segundo o relato 
de vivência por parte dos idosos, de situação emergencial relacionada à saúde no próprio domicílio estão representadas nas Tabelas 2 e 3, respectivamente.

Do total de idosos investigados, $56 \%$ avaliaram a situação de saúde vivenciada como negativa, sendo esta capaz de aumentar em duas vezes as chances de o indivíduo necessitar de atendimento emergencial no domicílio, quando comparado com idosos com autopercepção positiva de saúde. Em relação às limitações, $11 \%$ dos idosos foram impedidos de executar atividades habituais por problemas relacionados à própria saúde nas últimas duas semanas que antecederam a coleta de dados da PNS - 2013, 13\% apresentaram dificuldade na administração das próprias finanças, $24 \%$ em ir ao médico sozinho e $8 \%$ em sentar-se ou levantar-se da cadeira sem auxílio - limitações que elevaram em 1,66, 1,71, 2,10 e 1,67 vezes as chances de necessitarem de atendimento emergencial no domicílio.

Quanto à utilização de serviços de saúde, 22\% dos entrevistados relataram não procurar o mesmo médico ou serviço de saúde ao requerer atendimento de saúde, incrementando a chance em 1,34 de necessitar de atendimento emergencial no domicílio. Aumento das chances, de 1,39 e 7,02 vezes, respectivamente, deste mesmo evento ocorrer, também pode ser verificado para $30 \%$ dos idosos que realizaram mais de quatro consultas médicas em 12 meses, e para os 10\% que passaram por algum período de internação hospitalar considerando os 12 meses antecedentes à coleta de dados.

Tabela 2 - Análise descritiva das variáveis independentes que apresentaram relação com a necessidade de atendimento de emergência domiciliar de idosos brasileiros, Brasil, 2013.

\begin{tabular}{|c|c|c|c|c|c|c|c|}
\hline \multirow{2}{*}{ Variáveis dependentes } & \multirow{2}{*}{ Classe } & \multicolumn{2}{|l|}{ Total } & \multicolumn{2}{|l|}{ Sim } & \multicolumn{2}{|l|}{ Não } \\
\hline & & $\mathrm{n}$ & $\%$ & $\mathrm{n}$ & $\%$ & $\mathrm{n}$ & $\%$ \\
\hline Emergência domiciliar & & 23.815 & 100 & 675 & 3 & 23.140 & 97 \\
\hline \multirow{2}{*}{ Variáveis independentes } & \multirow{2}{*}{ Classe } & \multicolumn{2}{|l|}{ Total } & \multicolumn{2}{|l|}{ Sim } & \multicolumn{2}{|l|}{ Não } \\
\hline & & $\mathrm{n}$ & $\%$ & $\mathrm{n}$ & $\%$ & $\mathrm{n}$ & $\%$ \\
\hline \multirow{2}{*}{$\begin{array}{l}\text { Autopercepção de condição } \\
\text { de saúde geral }\end{array}$} & Positiva & 10.461 & 44 & 124 & 1 & 10.337 & 99 \\
\hline & Negativa & 13.354 & 56 & 551 & 4 & 12.803 & 96 \\
\hline \multirow{2}{*}{$\begin{array}{l}\text { Deixou de realizar quaisquer } \\
\text { de suas atividades habituais } \\
\text { por motivo de saúde } \\
\text { considerando as duas } \\
\text { últimas semanas }\end{array}$} & Não & 21.141 & 89 & 424 & 2 & 20.717 & 98 \\
\hline & Sim & 2.674 & 11 & 251 & 9 & 2.423 & 91 \\
\hline
\end{tabular}




\begin{tabular}{|c|c|c|c|c|c|c|c|}
\hline Variáveis independentes & Classe & Total & & Sim & & Não & \\
\hline $\begin{array}{l}\text { Dificuldade em administrar } \\
\text { as finanças sozinho }\end{array}$ & $\begin{array}{l}\text { Não } \\
\text { Sim }\end{array}$ & $\begin{array}{l}20.735 \\
3.080\end{array}$ & $\begin{array}{l}87 \\
13\end{array}$ & $\begin{array}{l}375 \\
300\end{array}$ & $\begin{array}{l}2 \\
10\end{array}$ & $\begin{array}{l}20.360 \\
2.780\end{array}$ & $\begin{array}{l}98 \\
90\end{array}$ \\
\hline $\begin{array}{l}\text { Dificuldade para ir ao } \\
\text { médico sozinho }\end{array}$ & $\begin{array}{l}\text { Não } \\
\text { Sim }\end{array}$ & $\begin{array}{l}18.146 \\
5.669\end{array}$ & $\begin{array}{l}76 \\
24\end{array}$ & $\begin{array}{l}239 \\
436\end{array}$ & $\begin{array}{l}1 \\
8\end{array}$ & $\begin{array}{l}17.907 \\
5.233\end{array}$ & $\begin{array}{l}99 \\
92\end{array}$ \\
\hline $\begin{array}{l}\text { Dificuldade para sentar ou } \\
\text { levantar da cadeira sozinho }\end{array}$ & $\begin{array}{l}\text { Não } \\
\text { Sim }\end{array}$ & $\begin{array}{l}2.1876 \\
1.939\end{array}$ & $\begin{array}{l}92 \\
8\end{array}$ & $\begin{array}{l}434 \\
241\end{array}$ & $\begin{array}{l}2 \\
12\end{array}$ & $\begin{array}{l}21.442 \\
1.698\end{array}$ & $\begin{array}{l}98 \\
88\end{array}$ \\
\hline $\begin{array}{l}\text { Procura o mesmo lugar, } \\
\text { mesmo médico ou mesmo } \\
\text { serviço de saúde quando } \\
\text { precisa de atendimento de } \\
\text { saúde }\end{array}$ & $\begin{array}{l}\text { Sim } \\
\text { Não }\end{array}$ & $\begin{array}{l}18.567 \\
5.248\end{array}$ & $\begin{array}{l}78 \\
22\end{array}$ & $\begin{array}{l}554 \\
121\end{array}$ & $\begin{array}{l}3 \\
2\end{array}$ & $\begin{array}{l}18.013 \\
5.127\end{array}$ & $\begin{array}{l}97 \\
98\end{array}$ \\
\hline \multirow[t]{3}{*}{$\begin{array}{l}\text { Frequência de consulta } \\
\text { médica no último ano }\end{array}$} & $\begin{array}{l}\text { Até } 4 \text { consultas } \\
\text { anuais }\end{array}$ & 12.467 & 52 & 257 & 2 & 12.210 & 98 \\
\hline & $\begin{array}{l}\text { Mais de } 4 \text { consultas } \\
\text { anuais }\end{array}$ & 7.036 & 30 & 407 & 6 & 6.512 & 93 \\
\hline & Não responderam & 4.312 & 18 & - & - & - & - \\
\hline $\begin{array}{l}\text { Internação hospitalar nos } \\
\text { últimos } 12 \text { meses }\end{array}$ & $\begin{array}{l}\text { Não } \\
\text { Sim }\end{array}$ & $\begin{array}{l}21.438 \\
2.377\end{array}$ & $\begin{array}{l}90 \\
10\end{array}$ & $\begin{array}{l}333 \\
342\end{array}$ & $\begin{array}{l}2 \\
14\end{array}$ & $\begin{array}{l}21.105 \\
2.035\end{array}$ & $\begin{array}{l}98 \\
86\end{array}$ \\
\hline
\end{tabular}

Fonte: Elaborada pelos autores.

Tabela 3 - Razões de chances em relação a necessidade de atendimento domiciliar emergencial de idosos, segundo variáveis independentes.

\begin{tabular}{lc}
\hline Variável & Odds Ratio (OR) \\
\hline Autopercepção da condição de saúde geral & 1,00 \\
Positiva & 2,00 \\
Negativa & \\
Deixou de realizar quaisquer de suas atividades habituais & \\
por motivo de saúde & 1,00 \\
Não & 1,66 \\
Sim & \\
Dificuldade em administrar as finanças sozinho & 1,00 \\
Não & 1,71 \\
Sim & \\
Dificuldade para ir ao médico sozinho & 1,00 \\
Não & 2,10
\end{tabular}




\begin{tabular}{lc}
\hline Variável & Odds Ratio (OR) \\
\hline Dificuldade para sentar ou levantar da cadeira sozinho & 1,00 \\
Não & 1,67 \\
Sim & \\
Procura o mesmo lugar, mesmo médico ou mesmo serviço & \\
de saúde quando precisa de atendimento de saúde & 1,00 \\
Sim & 1,34 \\
Não & \\
Frequência de consulta médica no último ano & 1,00 \\
Até 4 consultas anuais & 1,39 \\
Mais de 4 consultas anuais & \\
Internação hospitalar nos últimos 12 meses & 1,00 \\
Não & 7,02 \\
Sim &
\end{tabular}

Fonte: Elaborada pelos autores.

\section{Discussão}

Os achados demonstram que cerca de 3\% dos idosos participantes da PNS - 2013 confirmaram a vivência de alguma situação de emergência domiciliar, considerando o período de 12 meses antecedentes à coleta de dados, e que 8 das 56 variáveis independentes analisadas apresentaram forte relação com a variável desfecho. Em relação à autopercepção de saúde, quando o indivíduo percebe-se em uma condição negativa, este tem 2,00 vezes mais probabilidade de vivenciar uma situação de emergência domiciliar. Além desse indicador trazido pelo presente estudo, a condição de saúde percebida como negativa pode prognosticar quadros de declínio funcional, ocorrência de dependência física, hospitalizações e influenciar diretamente taxas de mortalidade entre idosos (JARDIM; BARRETO; GIATTI, 2010; SILVA; PINTO JÚNIOR; VILELA, 2014; JEREZ-ROIG et al., 2016; BELÉM et al., 2016).

Como exposto na Tabela 3, indivíduos que por algum motivo de saúde foram impedidos de realizar atividades pertinentes à rotina diária têm 1,66 mais probabilidade de vivenciar um episódio de emergência domiciliar quando em comparação com idosos que não foram inviabilizados de executar tais atividades. A fragilidade imposta pela presença de uma ou mais doenças ao longo dos anos, somada às circunstâncias decorrentes do processo natural de envelhecimento, contribui diretamente com a debilidade física, afeta as relações sociais, influencia negativamente aspectos culturais e econômicos (NUNES 
et al., 2017; BRITO; MENEZES; OLINDA, 2016), transformando a qualidade de vida de idosos, família e comunidade. Além disso, quando o idoso expõe uma autopercepção de saúde ruim, há um incremento nos seus níveis de incapacidade funcional (BELÉM et al., 2016; JEREZ-ROIG et al., 2016), fato que demonstra ligação direta com as demais variáveis independentes relacionadas à ocorrência de situações de emergência domiciliar.

A dificuldade funcional é um processo dinâmico e progressivo, influenciado por fatores multidimensionais (NUNES et al., 2017), com destaque para as questões genéticas e as circunstâncias vivenciadas pelo indivíduo (PEREIRA et al., 2017; VIRTUOSO JUNIOR et al., 2016). Como forma organizativa e classificatória para as dificuldades funcionais do indivíduo, a literatura apresenta comumente a divisão em duas categorias, as quais são relacionadas entre si: Atividades Básicas de Vida Diária (ABVD) e Atividades Instrumentais de Vida Diária (AIVD). Enquanto o primeiro agrupamento refere-se a atividades condizentes com autocuidado e desempenho motor, considerando a realização das rotinas diárias, o segundo grupo envolve a resolução das atividades atreladas à vida na comunidade e ao desempenho cognitivo (NUNES et al., 2017; PEREIRA et al., 2017; CAMPOS et al., 2016).

A presente investigação apontou a relação de uma variável do grupo de ABVD (dificuldade para sentar ou levantar da cadeira sozinho) e de duas variáveis do grupo de AIVD (dificuldade em administrar as finanças sozinho; dificuldade para ir ao médico sozinho) com a ocorrência de emergências domiciliares. As chances de vivenciar tais situações elevaram-se $\mathrm{OR}=1,67$, $\mathrm{OR}=1,71$ e $\mathrm{OR}=2,10$, respectivamente, para indivíduos que apresentaram tais restrições, quando comparados com os que não as tiveram.

Os estudos publicados arrolados a emergências domiciliares protagonizadas por idosos relacionam-se principalmente à divulgação de características epidemiológicas, elucidando a causa ou a situação problema, e são, em sua grande maioria, estudos transversais, o que dificulta a problematização em relação ao quadro prévio e o ocorrido (TENÓRIO; CAMACHO, 2015; RODRIGUES; RIBEIRO, 2012). Contudo, infere-se que dificuldades relacionadas à coordenação motora, ao equilíbrio, ao desenvolvimento de atividades habituais, ao autocuidado, entre outras, quando exibidas previamente, levam à dependência e expõem o indivíduo a maior risco de quedas, fraturas e acidentes domésticos (NASCIMENTO; TAVARES, 2016), aumentando a suscetibilidade a episódios emergenciais no domicílio, assim como à utilização da rede de serviços de urgência e emergência (LOPES et al., 2015). Outra hipótese é a de que dificuldades funcionais instauradas conduzam idosos a acessarem serviços de emergência no próprio domicílio, por considerarem estes mais 
ágeis e resolutivos para o tratamento clínico necessário no momento (SERBIM; GONÇALVES; PASKULIN, 2013).

Em relação a outro resultado encontrado, o qual aponta que indivíduos que não buscam um mesmo serviço médico quando requerem atendimento de saúde têm 1,34 vezes mais chances de sofrerem uma emergência domiciliar, consideram-se duas hipóteses. A primeira é a de que a busca por diferentes serviços possa estar relacionada com o maior grau de morbidade ou comorbidades entre os idosos, tendo em vista o acolhimento de demandas específicas de saúde e que não são supridas por profissionais que rotineiramente prestam o cuidado; e a segunda, que a própria situação emergencial induza à busca por serviços de saúde diferentes dos comumente utilizados (GOMIDE; PINTO; FIGUEIREDO, 2012). A literatura aponta que o acompanhamento rotineiro em um mesmo serviço de saúde, e com os mesmos profissionais, contribui no processo do cuidar de forma integral, com qualidade e resolutividade, além de encorajar o vínculo e a confiança entre os envolvidos, principalmente em serviços de nível primário (COSTA; CIOSAK, 2010; GOMIDE; PINTO; FIGUEIREDO, 2012).

Indivíduos idosos, quando comparados a sujeitos mais jovens, são associados a taxas superiores de hospitalizações, além de corresponderem a períodos prolongados de permanência e maiores índices de (re)internação (QUEIROZ et al., 2016; NUNES et al., 2017). Esses números elevam-se também quando os idosos possuem alguma limitação funcional, buscam mais por serviços de saúde e consultas médicas, e possuem diagnóstico médico de doença crônica (FIALHO et al., 2014). Contudo, os dados encontrados no presente estudo não evidenciaram tendências associativas entre episódios de emergência domiciliar e o avançar da idade.

As consultas médicas são medidas oportunas e efetivas para diagnósticos precoces e suas deliberações são ferramentas para ações de promoção e prevenção em saúde. Entretanto, quando a demanda é intensa, considerando um mesmo paciente e um curto período de tempo, o fato pode predizer a baixa resolutividade dos serviços de saúde (SILVA et al., 2017). A busca elevada por consultas médicas, considerando o período de 12 meses, e o número maior do que quatro intervenções, além de se relacionar com quadros de dificuldade funcional (FIALHO et al., 2014), está associada a indivíduos que se autopercebem em uma situação ruim de saúde (SILVA et al., 2017; BELÉM et al., 2016), indivíduos com amparo familiar reduzido ou inexistente, ou ainda portadores de plano de saúde (ALMEIDA, 2015). Compreende-se que idosos submetidos a elevadas consultas médicas repetidamente e internações hospitalares frequentes encontram-se em circunstâncias de saúde vulneráveis, e protagonizam 
situações de emergência domiciliar com maior frequência (CALDAS et al., 2015) quando comparados a idosos de mesma faixa etária livres de tais condições.

Considerando as variáveis que tiveram relação com situações de emergência domiciliar envolvendo idosos, cabe a reflexão em relação à demanda e oportunização dos serviços de saúde. Nitidamente, é possível visualizar a importância do acompanhamento preventivo de saúde de forma rotineira para indivíduos idosos, buscando preservar ou aprimorar suas capacidades funcionais nos níveis básicos e instrumentais, diminuindo a exacerbada necessidade de serviços avançados de saúde e de internações hospitalares, culminando em melhores indicadores de qualidade de vida e de autopercepção de saúde, assim como na otimização da rede de serviços de saúde.

As situações indicadas neste estudo remetem para a real necessidade de instrumentalizar familiares e agentes cuidadores de idosos para a vivência e atuação direta frente a episódios de emergência domiciliar, e para a necessidade de vigilância constante e conhecimento para executar o cuidado. Na incessante jornada por serviços de saúde resolutivos que prezem pela qualidade e que direcionem suas ações pautando-se no amplo conceito de saúde, destacam-se a Rede de Atenção Domiciliar (BRASIL, 2016) e a Rede de Atenção às Urgências e Emergências (BRASIL, 2011), cujas propostas contornam grande parte do trajeto percorrido no presente estudo, em especial no que tange aos campos de capacitação do próprio indivíduo e de familiares, com vistas à criação de uma base de apoio e garantia do cuidado do indivíduo idoso.

Finalmente, alerta-se, para a capacitação dos profissionais atuantes nos serviços de atendimento, a urgência e a emergência, para que estes possam identificar precocemente situações agudas e evitar ou reduzir prováveis complicações e para a otimização da gestão do cuidado, com a consolidação de protocolos de cuidado à saúde e de organização do serviço. Uma parcela dos dados da base provinda da PNS - 2013, relacionada aos indivíduos com 60 anos ou mais, foi fornecida por terceiros (morador do domicílio), e não necessariamente o próprio idoso. Todavia, já foram publicados estudos que comprovam a estabilidade dos dados em situações semelhantes, quando na ocorrência de outro respondente (FERREIRA; MATOS; LOYOLA FILHO, 2015).

Além disso, as informações prestadas pelos respondentes, em especial no que tange à utilização de serviços de saúde, estão sujeitas a viés de memória. No entanto, considerando a magnitude do evento emergência hospitalar na vida das pessoas, a probabilidade desse viés ocorrer torna-se menor. 
A pesquisa demonstrou a influência de aspectos múltiplos sobre um episódio de emergência domiciliar entre idosos, sendo que os que apresentaram maior relação foram a autopercepção negativa de saúde, a situação de internação hospitalar, determinadas limitações da vida diária e a falta de constância a um mesmo serviço de saúde. Considerando o incremento mundial da população idosa e de suas demandas de saúde, a interpretação dos presentes achados compõe uma importante base para o planejamento e desenvolvimento de ações preventivas e promotoras de saúde voltadas a idosos, além de estimular a capacitação de cuidadores e profissionais de saúde (ou em formação). Ainda, os resultados sugerem investimentos em atividades que provoquem a manutenção de capacidades físicas e psíquicas em idosos, seu empoderamento e protagonismo em saúde.

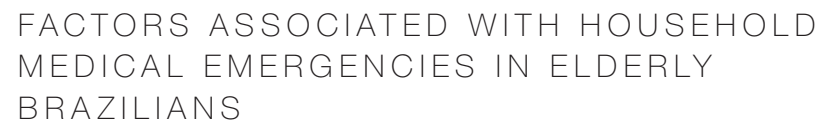

The objective of the study is to analyze the factors associated with the experience of household emergencies by Brazilian elderly. Cross-sectional, quantitative study with secondary data source - National Health Survey - 2013. The sample consisted of individuals aged 60 years or over $(n=23,815)$. The outcome variable was named "Household emergency", 56 independent variables were included. The data were processed, then the dimensionality reduction was performed in the Waikato Environment for Knowledge Analysis software. The variables strongly associated with home emergency situations were analyzed through logistic regression, to measure the intensity of the connection. The findings indicate that home emergency situations involving the elderly are more likely to occur when the individual had to be hospitalized in the last 12 months $(\mathrm{OR}=7.02)$, had difficulty going to the doctor alone $(O R=2.10)$, self-perceived negatively their health $(\mathrm{OR}=2.00)$, has difficulty managing their own finances $(O R=1.71)$, has difficulty sitting or getting up from a chair $(\mathrm{OR}=1.67)$, has failed to perform any usual activity $(\mathrm{OR}=1.66)$, had sought the same physician or health service when they needed care $(\mathrm{OR}=1.34)$ and had a frequency of more than four medical visits 

preventive and health promotion actions.

\title{
keywords
}

Health Systems. Health of the Elderly. Emergencies.

\author{
referências
}

ALMEIDA, Alexandre Nunes de. $\mathrm{O}$ acesso aos serviços de saúde pelos idosos no Brasil com base na Pesquisa Nacional por Amostra de Domicílios (PNAD) entre 1998 e 2008. Jornal Brasileiro de Economia da Saúde, São Paulo, v. 7, n. 1, p. 43-52, 2015.

ALMEIDA, Ana Paula Santana Coelho et al. Determinantes socioeconômicos do acesso a serviços de saúde em idosos: revisão sistemática. Revista de Saúde Pública, São Paulo, v. 51, p. 1-15, 2017.

BELÉM, Patrícia Leite de Oliveira et al. Autoavaliação do estado de saúde e fatores associados em idosos cadastrados na Estratégia Saúde da Família de Campina Grande, Paraíba. Revista Brasileira de Geriatria e Gerontologia, Rio de Janeiro, v. 19, n. 2, p. 265-276, jan./abr. 2016.

BONATO, Vera Lucia. Gestão de qualidade em saúde: melhorando assistência ao cliente. Mundo da Saúde, São Paulo, v. 35, n. 3, p. 319-331, 2011

BRASIL. Ministério da Saúde. Gabinete do Ministro. Portaria n. ${ }^{\circ}$ 1.600, de 7 de julho de 2011. Reformula a Política Nacional de Atenção às Urgências e institui a Rede de Atenção às Urgências no Sistema Único de Saúde (SUS). Brasília, DF: Ministério da Saúde, 2011. Disponível em: <http://bvsms.saude.gov.br/bvs/saudelegis/gm/2011/ prt1600_07_07_2011.html>. Acesso em: 19 mar. 2018.

Ministério da Saúde. Gabinete do Ministro. Portaria n. ${ }^{\circ} 825$, de 25 de abri de 2016. Redefine a Atenção Domiciliar no âmbito do Sistema Único de Saúde (SUS) e atualiza as equipes habilitadas. Brasília, DF: Ministério da Saúde, 2016. Disponível em: <http://bvsms.saude.gov.br/bvs/saudelegis/gm/2016/prt0825_25_04_2016.html>. Acesso em: 25 mar. 2018.

BRITO, Kyonayra Quezia Duarte; MENEZES, Tarciana Nobre de; OLINDA, Ricardo Alves de. Incapacidade funcional: condições de saúde e prática de atividade física em idosos. Revista Brasileira de Enfermagem. Brasília, v. 69, n. 5, p. 825-832, set./out. 2016.

CALDAS, Celia Pereira et al. Atendimento de emergência e suas interfaces: o cuidado de curta duração a idosos. Jornal Brasileiro de Economia da Saúde, São Paulo, v. 7, n. 1, p. 62-69, 2015

CAMPOS, Ana Cristina Viana et al. Prevalence of functional incapacity by gender in elderly people in Brazil: a systematic review with meta-analysis. Revista Brasileira de Geriatria e Gerontologia, Rio de Janeiro, v. 19, n. 3, p. 545-559, May/June 2016

COSTA, Maria Fernanda Baeta Neves Alonso; CIOSAK, Suely Itsuko. Atenção integral na saúde do idoso no programa saúde da família: visão dos profissionais de saúde. Revista da Escola de Enfermagem, São Paulo, v. 44, n. 2, p. 437-444, jun. 2010

DAMACENA, Giseli Nogueira et al. O processo de desenvolvimento da Pesquisa Nacional de Saúde no Brasil, 2013. Epidemiologia e Serviços de Saúde, Brasília, v. 24, n. 2, p. 197-206, abr./jun. 2015 
FERREIRA, Daniela Nice; MATOS, Divane Leite; LOYOLA FILHO, Antônio Ignácio de. Absence of routine medical consultation among hypertensive and/or diabetic elders: an epidemiological study based on the Brazilian National Household Survey 2008. Revista Brasileira de Epidemiologia, São Paulo, v. 18, n. 3, p. 578-594, July/Sep. 2015

FIALHO, Camila Bruno et al. Capacidade funcional e uso de serviços de saúde por idosos da Região Metropolitana de Belo Horizonte, Minas Gerais, Brasil: um estudo de base populacional. Cadernos de Saúde Pública, Rio de Janeiro, v. 30, n. 3, p. 599-610, mar. 2014

GOMIDE, Mariana Figueiredo Souza; PINTO, lone Carvalho; FIGUEIREDO, Luana Alves de. Acessibilidade e demanda em uma Unidade de Pronto Atendimento: perspectiva do usuário. Acta Paulista de Enfermagem, São Paulo, v. 25, n. 2, p. 19-25, 2012.

HIRSHON, Jon Mark et al. Health systems and services: the role of acute care. Bulletin of the World Health Organization, Geneva, v. 91, n.1, p. 386-388, Sep. 2013.

INSTITUTO BRASILEIRO DE GEOGRAFIA E ESTATÍ́STICA (IBGE). Pesquisa Nacional de Saúde 2013: percepção do estado de saúde, estilo de vida e doenças crônicas. Rio de Janeiro, 2014. Disponível em: <ftp://ftp.ibge.gov.br/PNS/2013/pns2013.pdf>. Acesso em: 25 mar. 2018

JARDIM, Renata; BARRETO, Sandhi Maria; GIATTI, Luana. Auto-relato e relato de informante secundário na avaliação da saúde em idosos. Revista de Saúde Pública, São Paulo, v. 44, n. 6, p. 1120-1129, dez. 2010.

JEREZ-ROIG, Javier et al. Autopercepção da saúde em idosos institucionalizados. Ciência \& Saúde Coletiva, Rio de Janeiro, v. 21, n. 11, p. 3367-3375, nov. 2016.

LOPES, Maria Carolina Barbosa Teixeira et al. Factors associated with functional impairment of elderly patients in the emergency departments. Einstein, São Paulo, v. 13, n. 2, p. 209-214, Apr./June 2015.

NASCIMENTO, Janaína Santos; TAVARES, Darlene Mara dos Santos. Prevalência e fatores associados a quedas em idosos. Texto \& Contexto Enfermagem, Florianópolis, v. 25, n. 2, p. 1-9, jun. 2016

NUNES, Juliana Damasceno et al. Indicadores de incapacidade funcional e fatores associados em idosos: estudo de base populacional em Bagé, Rio Grande do Sul Epidemiologia e Serviços de Saúde, Brasília, v. 26, n. 2, p. 295-304, abr./jun. 2017.

PASKULIN, Lisiane Manganelli Girardi; VALER, Daiany Borghetti; VIANNA, Lucila Amara Carneiro. Utilização e acesso de idosos a serviços de atenção básica em Porto Alegre (RS, Brasil). Ciência \& Saúde Coletiva, Rio de Janeiro, v. 16, n. 6, p. 2935-2944, jun. 2011

PEREIRA, Livia Carvalho et al. Fatores preditores para incapacidade funcional de idosos atendidos na atenção básica. Revista Brasileira de Enfermagem, Brasília, v. 70, n. 1 , p. 112-118, jan./fev. 2017.

QUEIROZ, Daiane Borges et al. Perfil de internações de idosos em uma clínica de neurociências de um hospital público. Revista Enfermagem Contemporânea, Salvador, v. 5, n. 1, p. 16-24, jan./jun. 2016.

RODRIGUES, Camilla Christina; RIBEIRO, Rita de Cássia Helú Mendonça. Perfil epidemiológico dos idosos atendidos na emergência de um hospital escola. Arquivos de Ciência e Saúde, São José do Rio Preto, v. 19, n. 2, p. 37-41, abr./jun. 2012.

SERBIM, Andreivna Kharenine; GONÇALVES, Ana Valéria Furquim; PASKULIN, Lisiane Manganelli Girardi. Caracterização sociodemográfica, de saúde e apoio social de idosos usuários de um serviço de emergência. Revista Gaúcha de Enfermagem, Porto Alegre, v. 34, n. 1, p. 55-63, mar. 2013.

SILVA, Alexandre Moreira de Melo et al. Use of health services by Brazilian older adults with and without functional limitation. Revista de Saúde Pública, São Paulo, v. 51, n. 1, p. 1-9, jun. 2017. 
SILVA, Isnanda Tarciara da; PINTO JUNIOR, Elzo Pereira; VILELA, Alba Benemérita Alves, Autopercepção de saúde de idosos que vivem em estado de corresidência. Revista Brasileira de Geriatria e Gerontologia, Rio de Janeiro, v. 17, n. 2, p. 275-287, 2014.

SOARES, Flaviana Dávila de Sousa et al. Características dos atendimentos de idosos realizados em casos de urgência e emergência. Revista Interdisciplinar em Saúde, Cajazeiras, v. 3, n. 1, p. 129-147, jan./mar. 2016.

SOUZA-JÚNIOR, Paulo Roberto Borges de et al. Desenho da amostra da Pesquisa Nacional de Saúde 2013. Epidemiologia e Serviços de Saúde, Brasília, v. 24, n. 2, p. 207-216, abr./jun. 2015.

SZWARCWALD, Célia Landmann et al. Pesquisa Nacional de Saúde no Brasil: concepção e metodologia de aplicação. Ciência \& Saúde Coletiva, Rio de Janeiro, v. 19, n. 2, p. 333-342, fev. 2014

TENÓRIO, Danielle Moura; CAMACHO, Alessandra Conceição Leite Funchal. Identificação dos agravos de saúde que levam os idosos ao serviço de emergência. Revista de Enfermagem UFPE On Line, Recife, v. 9, n. 1, p. 457-465, jan. 2015.

VIRTUOSO JUNIOR, Jair Sindra et al. Fatores associados à incapacidade funcional em idosos brasileiros. Revista Andaluza de Medicina del Deporte, Sevilla, v. 1, p. 1-7, set. 2016. Disponível em: <https://www.sciencedirect.com/science/article/pii/ S1888754616300867?via\%3Dihub>. Acesso em: 25 mar. 2018.

WITTEN, lan et al. Data mining: practical machine learning tools and techniques. 4. ed. Amsterdam: Morgan Kaufmann, 2016.

Data de Submissão: 25/05/2018

Data de Aprovação: 07/10/2018 